Dernière version avant épreuves, pour les citations, merci d'utiliser la version en ligne sur le site de la revue ou sur cairn.

Girel Mathias, "Éthique de la croyance, scepticisme et pratique. À partir de William Kingdon Clifford ", Revue française d'éthique appliquée, 2019/2 (N8), p. 32-46. DOI :

10.3917/rfeap.008.0032. URL : https://www.cairn.info/revue-francaise-d-ethique-appliquee2019-2-page-32.htm

\title{
Éthique de la croyance, scepticisme et pratique. A partir de William Kingdon Clifford
}

Mathias Girel

ENS-PSL, USR République des Savoirs, Centre Cavaillès

Treat believing as a branch of gymnastics and there is nothing, however revolting, which you may not train yourself to swallow. (Leslie Stephen $\left.{ }^{1}\right)$

\section{Introduction}

On connaît généralement le mathématicien et philosophe William K. Clifford (1845-1879) à travers William James: La Volonté de croire (JAMES $(1897,2005)$ ) est une charge, incisive mais posthume, contre l'auteur de l'Éthique de la croyance ${ }^{2}$. Évoquant l'existence de questions non tranchées par l'état actuel de la science, James se faisait l'avocat d'un " risque » en matière de connaissance et voyait dans l'approche de la croyance de Clifford le vestige d'un scientisme étriqué, qui serait de toute part en train de s'effondrer :

Ne croyez rien, dit [Clifford, résumé par James], suspendez toujours votre jugement plutôt que d'encourir le risque terrible de croire ce qui est faux, et cela pour avoir accordé votre assentiment à des éléments de preuve [evidence] insuffisants33.

Quand il n'y a pas de preuves concluantes, peut-on adopter n’importe quelle branche d'une alternative, s'il y a un intérêt pratique à le

\footnotetext{
${ }^{1}$ STEPHEN $(1873,152)$.

2 JAmes $(2005,44)$. Pour l'Éthique de la croyance, article de 1877 , je suis l'anthologie de Madigan: ClifFord (1999), citée par la suite EoB. Voir, du même MADIGAN (2009). Une traduction a récemment été publiée dans GAULTIER (2018).
}

3 JAMES (2005., 53). 
faire, ce que semble penser James ? Ou doit-on simplement n'avoir pas d'opinion, ce que préfère Clifford ? Pour James, Clifford symbolise l'effet paralysant de la crainte de l'erreur, tel «un général proclam[ant] à ses soldats qu'il vaut mieux ne pas se battre que de risquer jamais la moindre blessure 4. " Aujourd'hui encore, les deux philosophes fournissent des repères, diamétralement opposés, dans le débat sur l'éthique de la croyance 5 . Clifford représenterait une face «fondationnaliste », " justificationniste », alors que James en serait l'envers décisionniste ${ }^{6}$. La plupart du temps, Clifford illustre également une transition insensible entre le justificationnisme et le scepticisme7.

S'il est certain que Clifford s'expose à ces lectures et qu'il caricature parfois lui-même ses propres idées, il reste que James a sérieusement altéré les idées de Clifford $^{8}$, car la maxime toujours citée - «On a tort, toujours, partout et pour qui que ce soit, de croire quoi que ce soit sur la base d'éléments de preuve insuffisants » $(\mathrm{EoB}, 77)$ - ne représente qu'un aspect de la position de ce dernier. Après un bref rappel des exemples de Clifford, dans la première section, nous tenterons de rendre à ce dernier le concept de croyance qui est le sien, ce qui devrait déjà faire apparaître suffisamment les simplifications de James. Il s'agira de mettre en doute l'idée que l'éthique de la croyance du savant anglais serait auto-réfutante et ne laisserait rien subsister de nos croyances, théoriques et pratiques. En effet, la position de Clifford serait pour ses critiques lourde d'un scepticisme latent, au sens où il faudrait finalement douter de tout ce que l'on ne peut justifier. L'article étudie trois niveaux d'objections à la thèse de Clifford, qui correspondent à autant de contresens à son égard : on pourrait y voir (a) un mixte instable entre conséquentialisme et universalisme : Clifford semble à la fois préoccupé par les conséquences de nos croyances et un devoir universel de les justifier (deuxième section) ; (b) une contradiction entre sa maxime et les développements les plus prometteurs de la science : à ce compte, elle ne semble pas même laisser subsister la doctrine évolutionniste sur laquelle des doutes restent possibles au moment où il écrit (troisième section); et enfin (c) une contradiction avec nos croyances pratiques : la maxime ne se retourne-telle pas contre les notions de bien et de mal, pour lesquelles nous manquons d'evidence, ce qui compromettrait à sa racine l'idée d'une

\footnotetext{
4 JAMES $(2005,54)$.

5 Voir, entre de nombreux autres, Aikin (2014), FELDMAn (2006) et, ici, EnGEL (2001), PouiveT (2003), BOUVERESSE (2007).
}

6 Voir par exemple ENGEL $(2001, \S 2)$.

7 Voir JAMES $(2005,53)$.

8 Voir en premier lieu HOLLINGER (1997). 
éthique de la croyance? (Section 4) Ne se retourne-t-elle pas contre la plupart de nos croyances " naturelles » que nous serions bien en peine de justifier? (Sections 5 et 6)

\section{Expériences de pensée}

Le texte de Clifford est construit autour de deux exemples :

(1) Le premier exemple est celui de l'armateur, qui, malgré les doutes qui lui sont suggérés, parvient à croire que son bateau «tiendra » la mer, sans avoir pris toutes les précautions pour le vérifier, et qui envoie ses passagers au naufrage 9 . L'armateur surmonte toutes les raisons de douter qui semblent s’imposer à lui, il les « fait taire » ou parvient à leur opposer des raisons spécieuses (l'expérience passée, le sérieux habituel des fabricants de bateaux, la Providence qui serait vraiment injuste si elle faisait couler un bateau rempli d'immigrants), raisons qui lui permettent de ne pas considérer les risques, voire de les escamoter. L'armateur a bien une croyance, il ne se contente pas de tout ignorer de l'état de son bateau ou d'y être totalement indifférent, mais cette croyance, aussi stable soitelle, n'est pas fondée sur des éléments qui lui permettent d'affirmer que le bateau est sûr et peut prendre la mer.

(2) Le second exemple concerne une communauté accusée, à tort, par un petit groupe d'agitateurs zélés, d'user de moyens déloyaux pour ne pas avoir à enseigner à ses enfants les principes de la « vraie » religion. Ici encore, si les agitateurs qui en appellent à la vindicte sur cette petite communauté l'avaient voulu, ils auraient sans peine pu trouver des indices montrant qu'elle n'était pas coupable des fautes dont on l'accuse. Les zélateurs ont bien une croyance sur les agissements de cette petite communauté, mais cette croyance repose finalement sur des éléments qui n'ont rien à voir avec ce que fait réellement ladite communauté. On pourra rendre les accusateurs responsables de cette croyance qu'ils ont professée et qu'ils n'avaient pourtant aucun titre à soutenir, faute de preuves, faute d'éléments à charge, faute d'evidence ${ }^{10}$.

Il n'y a jusque-là rien que de très classique et de très prévisible. On retrouve évidemment dans l'article ce qui a fait la célébrité de Clifford : on commet une faute autant qu'une erreur quand on adopte une croyance sur la base d'éléments insuffisants. Les croyances mal fondées peuvent évidemment, comme dans ses deux exemples, avoir des conséquences

\footnotetext{
${ }^{9}$ L'attaque à peine déguisée contre la religion n’a pas échappé aux lecteurs de Clifford.

${ }^{10}$ Le terme anglais evidence, omniprésent dans la littérature, est délicat à traduire. On se rabat en général sur « éléments de preuve ».
} 
directes désastreuses : les émigrants font naufrage, la petite communauté est persécutée. On s'attend à ce que Clifford dise que la croyance adoptée est mauvaise ou illégitime en raison de ces conséquences directes. Mais ce n'est pas le cas : Clifford estime, dans une seconde expérience de pensée, que, même si le bateau arrive finalement à bon port, même si la petite communauté était finalement, après examen, coupable des pires turpitudes, l'armateur et les meneurs de cabale auront toujours mal agi.

\section{Conséquentialisme et universalisme.}

Le premier niveau d'objection consiste à dire que Clifford juge les croyances de deux points de vue différents, celui des conséquences et celui des raisons, mais un examen plus attentif montrera que cette opposition ne décrit pas assez précisément l'argument de Clifford.

On ne peut plus dans la deuxième expérience de pensée dire que ces croyances, celle de l'armateur, celle des « agitateurs », ont porté atteinte directement à leurs « objets ». Mais pour Clifford, cela ne les rend pas moins «condamnables". Sans doute faut-il prendre quelques précautions : il faut noter, comme le fait Susan Haack ${ }^{11}$, que dans le texte sur l'éthique de la croyance, Clifford ne se risque pas à se prononcer sur tel ou tel type de rapport entre les normes épistémiques et les normes éthiques ${ }^{12}$. Il faut également garder à l'esprit l'avertissement du même auteur : " dans les débats célèbres entre James et Clifford, c'est leur échec commun à distinguer la justification éthique de la justification épistémique qui crée la fausse impression que l'on doit choisir entre soit l'analyse excessivement exigeante d'un point de vue moral de l'“Éthique de la croyance", soit l'analyse par trop laxiste épistémologiquement de la "Volonté de croire" »13. Ce qui nous fait hésiter à adopter jusqu'au bout cette interprétation est le fait que Clifford, qui a réfléchi à la question des normes éthiques, ne semble pas même admettre, comme on va le voir, qu'en ce domaine la « justification » ait grand rôle à jouer.

Une telle opposition, entre les conséquences et les raisons, a un autre défaut : elle ne fait pas assez apparaître l'accent mis par Clifford sur la méthode, un pragmatiste dirait : sur « l'enquête ». Le caractère « bon » ou " mauvais » d'une croyance, ici, dérive de la façon dont elle est obtenue ; elle ne saurait être jugée par les résultats particuliers. La vertu épistémique

\footnotetext{
11 НАACK $(1997,135-38)$.

12 Il est tentant de penser, comme Haack, qu'il adopte une thèse de corrélation plutôt que de chevauchement (overlap), voir HAACK $(1997,129)$.

13 Ibid., 130.
} 
des croyances provient tout entière de leur « origine », non de leur « matière » $(\mathrm{EoB}, 71)$.

La question du bien et du mal concerne l'origine de sa croyance, pas son objet (matter) ; non ce qu'elle était, mais comment [on] l'a obtenue; non la question de savoir si elle s'est révélée vraie ou fausse, mais si [on] avait le droit de croire sur la base des éléments de preuve (evidence) qui étaient devant soi. EoB, 71.

Il semble pourtant bien qu'il y ait là deux principes incompatibles : dans un premier cas, ce sont les conséquences néfastes qui rendaient l'armateur et les zélateurs condamnables, dans le second, c'est l'observation ou non d'un mystérieux devoir épistémique.

Un premier élément de réponse est le suivant : lorsque Clifford parle d'evidence, il ne parle pas d'un état de fait, d'un observable singulier qui viendrait confirmer ou non une prédiction, il parle de l'examen de faits à la lumière d'un mode de raisonnement, d'une méthode pour arriver à un résultat ${ }^{14}$. La preuve n'est pas tout entière dans la conclusion, et ce que Clifford appelle evidence est davantage le lien entre un observable et la méthode et le type d'arguments que tel ou tel résultat particulier. Il le dit à la fin de l'essai, au sujet de la loi d'Ohm : si l'humanité tout entière devait oublier la formule, mais que l'on sache quel problème on doit poser, quelles quantités on veut mettre en relation, on pourrait la retrouver « en une heure ». De même, l'armateur et les zélateurs agissent « mal » car ils acquièrent une croyance d'une façon incompatible et contradictoire avec la méthode qui permettrait d'acquérir une croyance vraie sur le même sujet ; l'issue du voyage, ou de la cabale, n'y changent rien. Si dans les deux cas les croyances sont "mauvaises", c'est parce que leur vérité n'est qu'accidentelle et que dans la plupart des cas elles nous tromperont.

Deuxièmement, il n'y a pas lieu ici d'opposer frontalement le conséquentialisme et l'universalisme car le but de Clifford n'est pas ici d'opposer les utilitaristes et, disons, les kantiens. Son intérêt est ailleurs : il s'agit de montrer que dans les deux cas, une disposition ( "crédule ») a été enracinée, et que dans les deux cas, la croyance mal obtenue aura de toute façon des conséquences en dehors de ce cas particulier et de l'individu particulier qui en est le porteur ${ }^{15}$. Si l'on veut maintenir le terme, il faudrait alors plutôt parler d'un conséquentialisme élargi : l'adoption

14 Cf. EoB, 90-91.

15 Haack, dans l'article cité, s'oppose à Clifford sur ce point: elle estime qu'une nuisance potentielle n'est pas suffisante (car sinon on condamnerait les gens non seulement pour la conduite en état d'ivresse, mais aussi pour le simple fait de posséder une voiture). Elle estime également que le sujet n'est pas toujours responsable du fait de croire de façon injustifiée, dans le cas d'une « inadéquation cognitive » (Ibid.). Son argument sur les carences de la thèse de Clifford sont cependant trop elliptiques pour que l'on puisse ici les discuter plus en détail. Voir plutôt HELM $(1994,90-97)$. 
négligente a des conséquences sur la manière dont nous allons mener de futures enquêtes ou utiliser les enquêtes des autres. Mais l'opposition frontale dont nous parlions au départ ne tient pas parce que les conséquences portent sur la manière dont nous allons nous rapporter aux raisons.

Clifford a une philosophie de l'esprit qui accorde une place primordiale au changement, plus qu'aux « états " ( L'esprit change si constamment que nous ne le connaissons que par ses changements », EoB, 85). Ce qui est dissipé par ce point de vue, c'est alors l'illusion substantialiste du caractère :

La somme de toutes ces marques est précisément ce que nous appelons le caractère, qui est donc lui-même une histoire de toute la vie précédente de l'individu, qui subit par conséquent en permanence des ajouts, qui croît en permanence, qui est en permanence en état de changement ${ }^{16}$.

L'état instantané n'est alors qu'une abstraction par rapport à un processus qui le traverse et lui fournit sa véritable dynamique. De la même façon que dans l'exemple du " caractère », ici, la conséquence néfaste de la croyance mal assurée se lit dans l'ensemble des croyances à venir du sujet, dans son attitude générale par rapport à l'expérience: toute croyance a des effets « sur les actions de celui qui la nourrit » $(\mathrm{EoB}, 73)$. Clifford a une conception dispositionnaliste de la croyance dans tous ses écrits. Ce n'est pas par accident qu'il se réfère parfois à Bain, le psychologue écossais qui interprétait les croyances comme autant de dispositions à agir et qu'il connaissait personnellement ${ }^{17}$.

La croyance appartient à l'homme et à la conduite des affaires humaines : aucune croyance n'est réelle tant qu'elle ne guide pas nos actions, et ces actions même fournissent un test de sa vérité. $\mathrm{EoB}, 82$.

Plus que l'universalisme que certains propos de Clifford semblent trahir, c'est en fait un holisme de la conduite, qui fait qu'aucune de nos actions, aucune de nos croyances n'est indifférente : une fois adoptée

[Une croyance] s'insère dans l'agrégat de croyances qui est le lien entre la sensation et l'action à tout moment de nos vies, et qui sont ainsi organisées et compactées qu'aucune partie ne peut en être isolée du reste, mais que toute nouvelle addition modifie la structure du tout. EoB, 73.

C'est pour cela qu'il n'y a pas de différence de nature entre les deux versions des expériences de pensée : que l'accident, que la persécution, aient lieu ou non, les conséquences sur celui qui croit sont les mêmes.

16 CliffoRd $(1879,1901,82)$

17 Cf. FISCH $(1986,91))$. 
Cet argument, fondé sur la philosophie de l'esprit, conduit Clifford à estimer qu'il n'y a pas de croyance purement privée : " Aucune croyance, d'aucun homme, (...) n'est une affaire qui le concerne lui seul » (EoB, 73). L'éthique de la croyance ne consiste pas tant en devoirs envers soi-même, qu'en une responsabilité vis-à-vis des autres, même dans les délibérations que nous estimerions les plus privées et les plus particulières. Sur ces principes-là, on peut bien concevoir qu'il est mauvais, toujours et partout, de croire sur la base de preuves insuffisantes : il ne s'agit nullement d'un devoir privé, ni d'une obligation d'ordre théologico-moral, comme chez Locke, mais d'une discipline vis-à-vis de soi qui n'est nullement dissociable des revendications que pourraient formuler nos semblables ${ }^{18}$. C'est une position qui n'est au fond pas très éloignée de celle de James Mill19. La question de Clifford n'est pas celle qui consisterait à poser les hommes de science en "policiers » des croyances, elle consiste à se demander ce qui nous engage vis-à-vis des autres quand nous croyons quelque chose dans un apparent isolement. C'est sur ce terrain-là qu'il faut critiquer Clifford, s'il faut le faire, et c'est davantage dans ce domaine que son texte atteint les extrêmes les plus périlleux ${ }^{20}$. La croyance, dès lors que l'on admet comme Clifford qu'elle n'est jamais totalement du domaine privé, peut-elle se soustraire au questionnement éthique, en quelque sens que l'on entende ce questionnement?

\footnotetext{
18 Pour cette raison, parmi les différentes reconstructions de l'argument de Clifford que propose Richard Rorty, c'est la troisième qui nous semble la moins éloignée de la véritable thèse de l'auteur de l'Éthique de la croyance : « Une conception minimale analogue à celle de Clifford peut être résumée dans l'affirmation que, bien que vos émotions vous regardent seul, vos croyances regardent tout le monde. (...) Car croire est, de façon inhérente, un projet public : nous tous, les usagers du langage, y participons ensemble. Nous avons tous les uns envers les autres une responsabilité de ne rien croire qui ne puisse être justifié aux yeux du reste des nôtres. Etre rationnel, c'est soumettre ses croyances - toutes ses croyances - au jugement de ses pairs », Rorty, dans PuTNAM $(1997,88)$.
}

${ }^{19}$ Bain, dans sa biographie de James Mill, rend compte de l'article The Formation of Opinions (1826). Mill, après avoir rappelé qu'on ne peut nous louer ou nous blâmer pour quelque chose que nous ne contrôlons pas, insiste en revanche sur le "péché de croire sans raisons (evidence) » (BAIN $(1882,304))$. Le nœud éthico-épistémique est très clair : "L'habitude de former des opinions et d'agir à partir d'elles sans disposer d'éléments de preuve (evidence), est l'une des habitudes les plus immorales de l'esprit. (...) Comme nos opinions sont les [mères] de nos actions, être indifférent au sujet des éléments de preuve en faveur de nos opinions, c'est être indifférent quant aux conséquences de nos actions. Mais les conséquences de nos actions sont le bien et le mal de nos semblables. L'habitude de négliger les éléments de preuve

est donc l'habitude de négliger le bien et le mal de nos semblables. C'est une habitude de dureté et de cruauté, à grande échelle, et enracinée dans la partie la plus profonde de l'esprit » (cité par Bain, 305-306). On notera que par rapport aux textes de Locke, James Mill sécularise le « devoir » en question ; il ne s'agit plus d'un devoir vis-à-vis d'un créateur, mais vis-à-vis de ses semblables. De ce point de vue, le texte de Clifford ressemble beaucoup plus à celui de Mill qu'à celui de Locke.

20 Voir EoB, 75. 
Pour cette raison, aucune croyance n'est indifférente: toute croyance a forcément des effets dans l'action, sur l'ensemble de nos autres croyances qui régulent l'action, et dans les deux cas, sur les autres.

\section{L'éthique de la croyance nous force-t-elle à " douter " des derniers développements de la science?}

Il serait cependant envisageable que la maxime de Clifford se révèle impraticable, de son propre point de vue d' " homme de science », comme on disait alors. Lorsqu'un nouveau champ se développe, l'evidence est sujette à discussion. On pourrait notamment formuler l'argument selon lequel la théorie de l'évolution tombe elle-même sous le coup des interdits de l'éthique de la croyance, au moment où Clifford publie son texte. Estelle alors si prouvée que l'on ne puisse lui refuser son assentiment?

La maxime de Clifford réfuterait donc le socle théorique même sur lequel elle est bâtie, une vision de l'esprit revue à la lumière de l'évolution. Il est de ce point de vue intéressant de voir que Clifford a répondu luimême à cette question. L'article d'Avril 1878, Virchow on the Teachings of Science ${ }^{21}$ semble en effet être une telle contre-épreuve de l'éthique de la croyance: il s'agissait d'examiner la maxime de prudence de Virchow selon laquelle il ne fallait pas enseigner la doctrine de l'évolution tant qu'elle n'était pas davantage acceptée par la communauté des chercheurs. Cette précaution s'imposait selon Virchow tant que tous les points difficiles n'avaient pas été éclaircis, en particulier (1) l'émergence de l'organique à partir de l'inorganique et (2) l'origine de l'homme. La conférence de Virchow, âprement discutée dans tout le monde savant, avait une portée politique (il redoutait que la théorie évolutionniste ne serve de soutien aux thèses socialistes), et il exigeait finalement, ce qui fit beaucoup rire, que rien ne soit enseigné à l'école sur quoi on ne fût prêt à prêter serment. Autrement dit, Virchow semblait recommander un équivalent de la maxime de Clifford que l'on pourrait formuler ainsi : « Si les raisons de croire à la théorie de l'évolution sont insuffisantes, il est mauvais, partout et toujours, de faire de cette théorie un objet de croyance, et par là encore plus mauvais de l'enseigner aux enfants ». La réponse de Clifford comporte deux niveaux :

- Il répond tout d'abord à Virchow à partir des conséquences que celui-ci désignait. La menace de faire le lit du «socialisme » semble à

${ }^{21}$ Virchow (1821-1902), médecin et biologiste allemand. CLIFFORD (1879, 1901, 2, 298-342). Sur ce début qui fit rage pendant quelques mois, voir les références et le petit résumé dans PORTER (2004, 36-37). On pourra consulter avec profit le Popular Science Monthly, 1878, 13, 107-111. 
Clifford fantaisiste ( [En Angleterre], cela ne voudrait sans doute pas dire autre chose qu'une sorte d'alliance entre les boutiques coopératives et cette institution très respectable, le Metropolitan Board of works »22), et hors de propos.

- Il affronte en revanche plus en détail la difficulté théorique en admettant qu'avant d'enseigner n'importe quelle doctrine, il faut " attendre que la nature des éléments de preuve (evidence) qui la justifient puisse être comprise ${ }^{23}$. Cela veut dire que l'enseignant ne doit pas soumettre comme des dogmes l'idée de l'évolution et les résultats de cette théorie au sujet de telle ou telle espèce - les ongulés dans l'exemple de Clifford, car cela reviendrait en fait à faire la même chose que les créationnistes. Mais il peut en revanche attirer l'attention de ses élèves, dans les cours d'anatomie, sur la structure des extrémités (pieds, mains, doigts), qui est décisive dans de nombreux raisonnements mobilisés par la théorie de l'évolution. Le même raisonnement vaut pour les plans d'organisation. C'est une façon de les familiariser avec le type de preuves et de raisonnements qui sont nécessaires pour ensuite admettre la théorie de l'évolution. De la sorte, on évite de la proposer à leur croyance avant qu'ils ne disposent des moyens de comprendre les preuves qui poussent à l'admettre. C'est un raisonnement que Clifford étend aux considérations sur les atomes et sur les molécules.

La position de Clifford est donc tout le contraire du scepticisme que l'on a cru détecter chez lui, et elle n'exige en rien que l'on rompe avec la prudence qui est de mise à l'égard des hypothèses scientifiques. Il faut selon lui distinguer (1) un doute général sur notre propre capacité à connaître et (2) un doute qui s'appuierait sur des éléments précis de la doctrine à réfuter et dont on pourrait montrer qu'ils sont erronés ou mal fondés ; le premier remet en cause notre autonomie cognitive, le second est pleinement compatible avec l'attitude scientifique. Or, la critique formulée par Virchow lui semble plutôt exprimer le premier doute que le second. La théorie de l'évolution, au moment où il écrit, peut s'appuyer sur des démonstrations touchant d'autres espèces que l'homme ainsi que sur des recherches géologiques concordantes. Elle est, du point de vue de toutes les uniformités dont on dispose, moins invraisemblable que la création ex-nihilo ou que la genèse de l'homme directement à partir de l'inorganique. L'ennemi de l'éthique de la croyance que Clifford propose, c'est donc finalement le scepticisme, qui nous fait passer de l'un, le doute concret et reposant sur des raisons, à l'autre, le doute théorique :

22 ClifFord $(1879,1901,307-308)$

${ }^{23}$ Ibid., 324. 
Vous ne permettrez à aucune tentative malhonnête de vous convaincre de ne pas croire à la doctrine de l'évolution parce que Virchow a admis que certaines de ses parties ne sont pas encore absolument prouvées. C'est une chose que de croire qu'une doctrine est fausse, et tout à fait une autre d'admettre un doute théorique à son sujet. Je dis un doute théorique, parce que c'est un doute fondé sur l'imperfection nécessaire de toute connaissance humaine, et non sur quelque défaut pratique des données (evidence). Car un doute, précisément semblable en genre, quoique plus grand en degré, s'attache à l'énoncé que les Russes ont pris Pleven l'an dernier²4.

Si je défends un doute sous le prétexte que ma connaissance est imparfaite, j'en viendrai vite à formuler des doutes extravagants (à supposer que je suis un cerveau dans une cuve, que mon voisin a la tête remplie de sciure, que Pleven n'a pas été prise par les Russes), et j'aurai enfreint l'éthique de la croyance, car j'aurai admis une raison de douter sur la base de données ou de preuves insuffisantes. L'éthique de la croyance est dans le même temps une éthique du doute mesuré et limité par les raisons de douter.

De fait, on n'est jamais tenu d'avoir des croyances sur ce que l'on ne comprend pas, ce qui ne signifie pas que l'on dût en douter :

"Mais, dira-t-on, je suis une personne fort occupée ; je n'ai pas le temps pour ce long travail d'étude qui serait nécessaire pour me transformer le moins du monde en juge compétent au sujet de certaines questions, ou même de comprendre la nature des arguments." Cette personne ne devrait alors pas avoir le temps de croire. EoB, 78

\section{Éthique de la croyance et scepticisme}

Si donc la maxime de Clifford n'entre pas immédiatement en contradiction avec elle-même, ne menace-t-elle pas pour autant l'ensemble de nos croyances pratiques, ce qui la rendrait finalement aussi extravagante que ce qu'elle condamne? Pouvons-nous en effet justifier la totalité des croyances sur la base desquelles nous agissons? Clifford marque très nettement ce à quoi l'éthique de la croyance ne s'applique pas :

Courons-nous donc le danger de devenir des sceptiques universels, qui doutent de tout, qui redoutent toujours de mettre un pied devant l'autre tant que nous n'avons pas testé en personne la solidité de la route ? EoB, 78 .

Clifford envisage bien, pour les écarter, les objections que James croira devoir élever contre lui. Il y a ici deux dangers, solidaires, qui se profilent ici : après tout, si éthique il y a, il se trouve que nos notions du

${ }^{24}$ Ibid., 320. Allusion au siège de Plevna (Pleven), Juillet-Décembre 1877, épisode important de la guerre russo-turque. Douter de cet événement, au moment où Clifford écrit, n'est pas plus extravagant que supposer que «ces canailles de journaux ont tous ensemble conspiré pour tromper toute la nation britannique ». 
« bien » et du « mal » sont loin d'être les plus justifiables en raison ; par ailleurs, de très nombreuses croyances d'arrière-plan ne passent jamais sous l'œil de l'examen critique et il semble dangereux de formuler une éthique qui nous mettrait directement dans la situation de ne jamais pouvoir y obéir. L'éthique de la croyance serait donc foncièrement sceptique s'il s'agissait d'éprouver toute croyance de cette façon, en la soumettant au « devoir de l'enquête ».

Examinons le premier des deux points. « Bien », « mal », ne sont pas des notions dont le contenu serait fixé clairement une fois pour toutes ${ }^{25}$. Clifford défend ici une forme de darwinisme, au sens où il estime que la conscience morale est une fonction de la préservation de la société, dans sa lutte pour l'existence, mais on simplifie son propos quand on lui impute un sophisme naturaliste, qui consisterait à passer du « est » (donné par la théorie de l'évolution au « doit » (qui préciserait ce que nous devons faire pour que la société survive bien et mieux). Si je devais attendre de disposer d'une connaissance de la société pour juger bon tel ou tel motif, il y aurait effectivement là un cercle vicieux, mais Clifford ne pense nullement qu'à un moment donné, il y ait une telle indécision dans les termes de «bien » et de « mal » (dont il dit que ce sont des « platitudes » ${ }^{26}$ ). Nous ne jugeons pas telle ou telle chose bonne parce que nous estimerions qu'elle est propice au bien de la société. Nous comprenons, en analysant les "platitudes » au sujet du bien et du mal, que, sans doute, des intérêts sociaux, grégaires, s'expriment en elles. En ce sens, il y a une naturalité de nos croyances au sujet du bien et du mal, que Clifford préfère faire remonter à notre statut d'animaux grégaires plutôt qu'à une posture solipsiste ou à un jugement d'ordre théologico-moral. Clifford relativise si peu cette part naturaliste qu'il est prêt à admettre, ce qui ferait sans doute frémir certains de ses défenseurs :

Aucune maxime ne peut être valide en tout temps et en tout lieu pour tous les êtres rationnels. (...) Une maxime valide pour nous peut seulement être valide pour des portions de la race humaine telles qu'elles sont pratiquement identiques à nousmêmes ${ }^{27}$.

La démarche est comme chez Nietzsche, dont il a parfois été rapproché, généalogique, elle est descriptive, et non pas prioritairement prescriptive.

Qu'en est-il alors du second point, sur les croyances que nous ne remettons pas en cause ? Pour écarter l'accusation de scepticisme, Clifford

\footnotetext{
${ }^{25}$ ClifFoRd $(1875,1999)$. Ce qui a permis à certains lecteurs de le rapprocher de Nietzsche.

26 CLIFFoRd (1875, 1999, 28-29).

27 Clifford (1879, 1901, 2, 279, « Cosmic emotion »).
} 
établit une distinction : les croyances que sa règle vise ne sont que celles qui peuvent être " renforcées », " instituées ». À la manière de Peirce plus tard, il estime que nos croyances les plus profondes ne sont pas ébranlées par le mouvement de l'enquête :

Il n'y a aucun danger pratique que de telles conséquences découlent jamais d'un soin scrupuleux et d'un tel contrôle de soi en matière de croyances. [...]. Les croyances au sujet du bien et du mal qui guident nos actions à l'égard des hommes en société, et les croyances au sujet de la nature physique qui guident nos actions à l'égard des corps animés et inanimés, ces croyances ne souffriront jamais du fait de l'enquête ; elles se débrouilleront toutes seules, sans être étayées par des "actes de foi", par la clameur de partisans à la solde ou la suppression d'éléments de preuve (evidence) contraires. EoB, 78-79.

L'éthique de la croyance n'intéresse qu'une voie médiane en matière de croyances, entre ce que l'on pourrait appeler le « dispositionnel » lourd, l'ensemble de croyances que même une procédure de doute acérée ne peut entamer que superficiellement, et l'hypothétique, qui peut justement fonctionner comme heuristique dans l'obtention de nouvelles croyances fondées, mais auquel il serait exagéré de dire que nous croyons, au sens où ce serait une règle vivante à l'œuvre dans notre conduite. Cette première précision n'est pas anodine : la pratique de l'enquête n'est possible et ne tire sa légitimité que parce qu'elle s'adosse à un noyau de croyances qui, elles, ne seront pas remises en cause par l'enquête, même si elles peuvent l'être à leur tour, dans une autre enquête à venir.

Même si l'éthique de la croyance ne menace par principe ni les croyances théoriques ni les croyances pratiques, on pourrait cependant élever à son endroit la même critique que celle que l'on fait en général à Descartes : à savoir qu'il n'y a finalement, même dans le champ de ce que nous avons appelé les croyances médianes, que très peu de croyances qui peuvent être entièrement justifiées de la façon qu'il indique, si bien que, même si l'on admettait le principe de l'éthique de la croyance, elle conduirait à un appauvrissement considérable de ce que nous appelons la connaissance. Clifford perçoit très bien, même avec les précisions qu'il vient de faire, le risque de scepticisme qu'induit sa première maxime : il le perçoit même tellement que c'est tout l'enjeu de l'article, dans ses sections II et III, que d'évaluer "Le poids de l'autorité », et "Les limites de l'inférence », sections qui, elles, ne sont que très rarement commentées. Que se passe-t-il quand nous ne disposons pas, individuellement ou ponctuellement, des preuves dont nous avons besoin? 


\section{Croyance et autorité}

La première solution d'extension du domaine des éléments de preuve est classique : c'est celle du témoignage. Or, lorsque nous avons affaire au témoignage d'autrui, comme l'avait déjà vu Hume dans son examen des miracles, il y a deux questions qui se posent: (1) est-il honnête ? (2) est-il possible que le témoin se trompe ? Clifford critique la confusion entre les deux questions: (1) la première rejoint plus généralement la question des effets de la croyance (quelqu'un croit-il suffisamment à ce qu'il dit pour que l'on forme à ce sujet une assurance suffisante ?) ; (2) la deuxième question concerne la justesse de la croyance. Clifford, comme Peirce plus tard, s'intéresse aux effets de la croyance quand il se trouve qu'elle est juste, et donc plutôt à la deuxième question. Inversement, c'est pour lui le travers des attitudes religieuses ${ }^{28}$, que de se concentrer sur la première question et de tout faire reposer sur l'honnêteté supposée du témoin, au détriment de la seconde. Ceux qui posent la question de la sincérité ou celle du « succès » d'une croyance ne font que brouiller les cartes :

Le caractère de Mahomet est une preuve excellente du fait qu'il était honnête et disait la vérité pour autant qu'il la connaissait ; mais cela ne prouve pas du tout qu'il savait quelle était la vérité. EoB, 81.

Mais Clifford doit alors affronter l'objection qu'il a lui-même rendue possible. Si croire, c'est être disposé à agir, ne peut-on pas dire que la croyance est prouvée par ses effets ? C'est pour lui l'occasion de préciser une distinction entre deux choses, tester l'objet de la croyance et tester l'attitude de croyance, qui est précisément la distinction qui sera gommée chez James :

\footnotetext{
Mais on pourrait objecter qu'accepter l'Islam comme système est précisément cette action qui est engendrée (prompted) par la croyance en la mission du prophète, et qui servira d'épreuve à sa vérité. Est-il possible de croire qu'un système qui a si bien réussi pût reposer sur une illusion ? Non seulement les saints en tant qu'individus ont trouvé la joie et la paix dans la croyance, et ont vérifié ces expériences spirituelles qui sont promises aux fidèles, mais des nations ont également été tirées de la sauvagerie, ou de la barbarie, vers un état social plus élevé. Nous disposons sûrement de la liberté de dire que l'on a agi sur la base de cette croyance, et qu'elle a été vérifiée. EoB, 82.
}

Si Clifford fait ici exactement le portrait de la position que défendra James, sa réponse, en revanche, est très différente : pour Clifford, ce qui a été testé, et qu'effectivement l'expérience confirme, c'est la "sagesse pratique » du prophète dans le domaine des affaires humaines; c'est

${ }^{28}$ Il ne prend que l'exemple de l'Islam et du bouddhisme, mais son opinion sur le christianisme ne fait guère de doute, voir EoB, 88. 
également le «confort » que donnent certaines croyances à certaines personnes, mais ce n'est pas la vérité de la croyance elle-même. Ce qui a été testé, c'est au fond l'efficacité d'une institution politique.

Cet argument critique pourrait sembler classique, mais il se complète d'une face positive qui l'est beaucoup moins, car Clifford n'entend pas mettre en doute tout type de témoignage. Ici encore, il ne professe pas tant un individualisme en matière de croyances qu'une forme de «holisme» bien compris, et peut-être même une théorie de la connaissance distribuée. La maxime de Clifford, précisée par lui-même, admet l'équivalent de l'argument d'autorité :

La règle qui devrait nous guider dans de tels cas est pour le moins simple et évidente : que le témoignage agrégé de nos voisins soit sujet aux mêmes conditions que le témoignage de n'importe lequel d'entre eux. A savoir que nous n'avons aucun droit de croire qu'une chose est vraie parce que tout le monde le dit, à moins qu'il n'y ait de bonnes raisons de croire qu'une certaine personne au moins a les moyens de savoir ce qui est vrai, et dit la vérité pour autant qu'elle la connaît. EoB, 8829.

La question du fondement des croyances, et de la légitimité qu'il peut y avoir à les adopter, n'est donc en rien une affaire privée ; nous avions déjà rencontré sa dimension sociale, mais on peut maintenant ajouter qu'elle est sociale, non pas au sens faible où elle serait partagée, mais au sens fort où elle est distribuée : il y a une division sociale du travail de vérification. Il ne s'agit pas de croire uniquement ce que je peux vérifier avec mes maigres moyens, mais ce dont je sais, avec une evidence raisonnable, que quelqu'un au moins peut le vérifier. C'est le « au moins un » qui fait toute la différence entre la philosophie scientifique, et son idéal de collaboration et la philosophie de « séminaire » : l'idée de Clifford est qu'il y a au moins une personne qui peut vérifier que cette bague est en or, alors qu'il n'est pas certain que ce soit le cas pour la «transmutation des espèces $\gg 30$.

Clifford a au fond en tête un argument fort simple, il veut faire entendre à ses lecteurs une différence entre :

${ }^{29}$ Je souligne. Une telle formule fait fortement penser à l'argument de la division sociale du travail linguistique développée par Putnam dans "La Signification de 'signification' » (PUTNAM $(1975,215-271)$ ), dans la mesure où l'argument putnamien implique que la vérification est socialement distribuée et n'est jamais à comprendre en un sens individualiste.

30 Cette position présente évidemment, d'un point de vue plus récent, un mixte intéressant d'internalisme et d'externalisme (il y a bien (a) une " contribution » de l'environnement, (b) la sortie d'un modèle omniscient de la signification, tout en maintenant l'exigence que la référence ne soit pas fixée que par les attitudes propositionnelles de nos semblables). 
- Accepter une proposition qui est admise par une communauté ou, plus rarement, un individu, dont la fonction même est ( $\left.a^{\prime}\right)$ de la maîtriser ou ( $\left.a^{\prime}\right)$ de pouvoir la mettre à l'épreuve.

- Accepter une proposition que personne ne peut justifier ( $b$ ') parce qu'elle est acceptée par d'autres personnes, ( $b$ ") ou parce que rien n'est jamais totalement fondé si bien que toute proposition, même la plus établie, suppose un "risque », ce qui autoriserait à « croire » même quand la justification fait défaut.

Les deux branches de (b) sont condamnées, la première étant circulaire, la seconde correspondant au « doute théorique » que Clifford a critiqué plus tôt. Le chimiste qui nous dit comment réaliser un alliage mérite d'être cru, car c'est justement son métier que de le savoir, et il ne serait pas inenvisageable que moi-même je l'apprenne. En revanche, s'il nous dit que tel atome d'oxygène a conservé un poids et une fréquence de vibration inchangés depuis l'origine de l'univers, autre exemple donné par Clifford, il propose un énoncé qui n'est sujet à aucune vérification humaine, et ne mérite donc pas d'être cru. Qu'une personne ait une conscience explicite de la règle qu'il suit ou qu'il applique de façon fiable aux yeux de la communauté, et donc que l'on se trouve dans la situation $a^{\prime}$ ou $a$ ", ne change pas grand-chose ici.

On peut alors considérer une objection, qui se pose sans doute plus clairement à nous qu'à Clifford, mais qui n'en est pas moins décisive et qui a été formulée par Van Harvey ${ }^{3}$. Si l'on reprend l'exemple de l'armateur, nous le jugeons coupable non pas en général mais parce que c'est une de ses obligations statutaires que d'inspecter les bateaux et de s'assurer de leur état (c'est une tâche qui lui incombe). Si nous remontons de la mort des immigrants à la responsabilité de l'accident, quoique nous fassions, nous aboutissons au bureau de l'armateur. Ce qui fait la culpabilité de l'armateur, ce n'est pas qu'il ait enfreint un impératif universel, mais qu'il ait manqué à ses obligations statutaires, sinon, il faudrait dire que les immigrants sont également coupables d'avoir cru sur une base insuffisante que le bateau pourrait prendre la mer. L'intérêt de concevoir les choses ainsi est que l'on n'a plus affaire à un phénomène général et indistinct: il y a une croyance spécifique, avec un type d'evidence spécifique, prévu par le rôle de l'armateur, et qui correspond à la nécessité pour lui de dire « je sais que ce bateau peut prendre la mer », et donc de pouvoir donner des raisons. Le point fort, dans cette critique, tient dans l'idée de détermination particulière des normes qui s'imposent à chacun dans la pratique de l'enquête, et qui sont relatives à la perspective sociale. On voit que l'objection de Van Harvey, dont toute l'ingéniosité était de dire que l'on n'avait jamais affaire qu'à des devoirs épistémiques, assortis de conditions précises, dépendant de la position sociale du sujet, est en

31 VAN HARVEY (1986), qui précise que sa critique est d'inspiration wittgensteinienne. 
grande partie entamée, si l'on accepte la lecture qui vient d'être donnée de Clifford, lorsqu'il envisage une division sociale du travail de vérification.

\section{Croyance et uniformité}

Il y a enfin un deuxième registre d'extension des croyances, souvent négligé par ceux qui mentionnent Clifford. Dans la mesure où une bonne part de nos croyances comprennent une dimension d'attente, sont tournées vers l'avenir, il est bien évident que se pose à leur égard le même problème que vis-à-vis de l'induction : on ne peut «prouver » que les uniformités que nous connaissons se perpétueront dans l'avenir. Or, c'est justement parce que, dès le départ, Clifford inscrit les croyances dans l'économie de l'action qu'il ne peut avoir endossé la thèse que lui prête James et avoir professé un justificationnisme extrême. Précisons bien ce point: presque toutes nos croyances pratiques dépassent «par définition » notre expérience :

Un peu de réflexion nous montrera que toutes les croyances, même les plus simples et les plus fondamentales, dépassent l'expérience quand on les considère comme des guides pour nos actions. [...] La question n'est par conséquent pas, « Pouvons-nous croire ce qui dépasse l'expérience ? », car cela est impliqué dans la nature même de la croyance; mais, "Jusqu'où et de quelle façon pouvons-nous ajouter à notre expérience en formant nos croyances ? » EoB, 92.

De plus, il y a de nombreux cas où c'est notre devoir que d'agir sur la base de probabilités, bien que les éléments de preuve ne soient pas à même de justifier notre croyance présente ; parce que c'est précisément au moyen d'une telle action, et par l'observation de ses conséquences, que l'on obtient les éléments de preuve qui peuvent justifier la croyance future. Si bien que nous n'avons pas lieu de craindre qu'une habitude d'enquête consciencieuse paralyse notre vie quotidienne. EoB, 79.

Il est remarquable que Clifford se pose, et réponde à, deux questions que le pragmatisme devra affronter : d'une part, si une croyance est un guide pour l'action, en quoi une croyance scientifique, par exemple notre croyance en la présence d'hydrogène dans le soleil ou en la vérité de tel récit historique, guide-t-elle notre action? D'autre part, pouvons-nous expérimenter sur ce que nous ne pouvons observer directement? La réponse de Clifford est que la croyance en une vérité non directement observable a des conséquences inférentielles sur des réalités manipulables et observables. Par exemple, le phénomène physique exploité par le spectroscope couvre une grande diversité de domaines : si le spectroscope n'est pas un instrument fiable pour nous révéler la composition du soleil ou la nature physique des protubérances solaires, grand problème de l'époque, alors il ne le sera pas non plus pour reconnaître, ici, sur Terre, les différents composants chimiques que nous pouvons manipuler (EoB, 94). Or, cette fiabilité peut être attestée dans ces derniers cas, ne serait-ce 
que par recoupement entre plusieurs méthodes d'analyse chimique (spectroscopie, poids spécifique, réactifs spécifiques). Le principe qui permet l'extension est ici celui de l'uniformité de la nature : mais quelle est alors la nature de notre croyance à l'uniformité de la nature ? La réponse de Clifford, telle qu'elle nous est précisée dans un autre texte, est alors non pas logique mais causale. Elle est littéralement empruntée à Bain :

Or, l'inférence dépend de la supposition de l'uniformité de la nature, et sur quoi cette dernière repose-t-elle ? Nous ne pouvons pas inférer ce qui est le fondement de toute inférence, mais, bien que je ne puisse vous donner une raison logique de le croire, je peux vous donner une explication physique du fait que nous le croyons tous. Nous croyons une chose quand nous sommes préparés à agir comme si elle était vraie. Mais si vous et moi n'avions pas agi habituellement sur la base de la supposition de l'uniformité de la nature à partir du moment où nous nous pouvions agir un tant soit peu, nous ne serions pas ici pour poser la question. La nature sélectionne pour la survie ces individus et ces races qui agissent comme si elle était uniforme, et c'est de là que provient que cette croyance s'est répandue peu à peu sur le monde civilisé. EoB, 94.

C'est finalement la même explication, causale et non logique, qui est donnée au problème des autres consciences.

\section{Conclusion}

Que nous apporte ce rapide examen de l'Éthique de la croyance? L'approche de Clifford comporte bien des présupposés massifs que l'on n'est nullement tenu d'accepter. C'est le cas notamment de l'idée qu'il n'y a rien de purement privé dans l'adoption de n'importe quelle croyance. Mais cette idée-là n'est-elle pas en germe dans la thèse selon laquelle toute croyance est une disposition à agir ? C'est également une question ouverte que de savoir si une croyance mal acquise a forcément les conséquences néfastes, directes ou indirectes, que lui prête Clifford. Il sera en revanche peut-être apparu que le champ de la maxime de Clifford était beaucoup plus restreint que ce que l'on estimait généralement : pour lui, elle ne s'applique nullement aux croyances « ordinaires ». Clifford a également bien vu que, du fait de leur nature dispositionnelle, le champ d'application des croyances dépassait par définition toujours ce qui les fondait. La question de Clifford est de savoir de quelles croyances nous pouvons être responsables, et ce que signifie exactement cette responsabilité. Sur le plan épistémologique, transiger sur l'éthique de la croyance conduit tout droit à enraciner des « vices » cognitifs, dont l'effet ne se limite nullement à l'instant et à la situation présente. Sur le plan éthique, qui est le plus évident, l'enracinement de dispositions crédules va à l'encontre de l'émancipation qu'il appelle de ses vœux. Pour autant, le même texte offre de robustes outils pour contrer les doutes superficiels, les «doutes de papier » dont parlera Peirce, et passer à côté des nuances introduites par 
Clifford serait se méprendre profondément sur l'esprit de ce texte fondateur.

\author{
Références
}

\title{
AIKIN, SCOTT
}

2014. Evidentialism and the Will to Believe, Londres, Continuum.

\section{Bain, Alexander}

1882. James Mill. A Biography, Londres, Longmans, Green, and co.

\section{BOUVERESSE, JACQUES}

2007. Peut-on ne pas croire? Sur la vérité, la croyance et la foi, Marseille, Agone.

CLIFFORD, W. K.

1999. Right and Wrong: The Scientific Ground of their Distinction, [1875], dans: The Ethics of Belief and other Essays, Amherst, Prometheus Books, p. 28-69.

\section{CLIFFORD, WiLLIAM KINGDON}

1901. Lectures and Essays, [1879], Londres, New York, Macmillan.

1999. The Ethics of Belief and other Essays, T. Madigan (dir.), Great books in philosophy, Amherst, N.Y., Prometheus Books.

ENGEL, PASCAL

2001. Sommes-nous responsables de nos croyances?, Université de Tous les savoirs, Qu'estce que la culture?, 6, p. 429-439.

\section{FELDMAN, RichaRd}

2006. Clifford's Principle and James's Options, Social Epistemology, 20, 1, p. 19-33.

\section{GAULTIER, BENOIT}

2018. L'Immoralité de la croyance religieuse: L'éthique de la croyance de William Clifford, suivi de La volonté de croire de William James, Marseille, Agone.

\section{HAACK, SUSAN}

1997. The Ethics of Belief Reconsidered, dans: L. E. Hahn (Dir.), The Philosophy of Roderick M. Chisholm, Chicago, Open Court, p. 129-144. .

\section{HeLm, PaUl}

1994. Belief Policies, New York, Cambridge University Press.

\section{HOLLINGER, DAVID A.}

1997. James, Clifford, and the Scientific Conscience, dans: R. A. Putnam (Dir.), The Cambridge Companion to William James, New York, Cambridge University Press, p. 69-83.

\section{JAMES, WILLIAM}

2005. La Volonté de croire, [1897], Paris, Les empêcheurs de penser en rond. Tr.fr. L. Moulin.

MADIGAN, TIMOTHY

2009. W. K. Clifford and the Ethics of Belief, Cambridge, Cambridge Scholars Publishing.

\section{PORTER, THEODORE M.}

2004. Karl Pearson : the scientific life in a statistical age, Princeton, Princeton University Press.

POUIVET, ROGER

2003. Qu'est-ce que croire?, Chemins Philosophiques, Paris, Vrin.

1975. Mind, language, and reality, Cambridge ; New York, Cambridge University Press. 
Putnam, Ruth AnNa (Dir.)

1997. The Cambridge Companion to William James, New York, Cambridge University Press.

STEPHEN, LESLIE

1873. Essays on freethinking and plainspeaking, Londres, Longmans, Green and co.

VAN HARVEY, A.

1986. The Ethics of Belief reconsidered, dans: G. D. McCarthy (Dir.), The Ethics of belief debate, Atlanta, Ga., Scholars Press, American Academy of Religion Academy series, vol p. 189-203. 